\title{
Molecular lines as tracers of Compton-thick AGN?`
}

\author{
I. Georgantopoulos ${ }^{1,2}$, E. Rovilos ${ }^{3}$, A. Akylas ${ }^{2}$, and E. Xilouris ${ }^{2}$ \\ 1 INAF-Osservatorio Astronomico di Bologna, via Ranzani 1, 40127 Bologna, Italy \\ e-mail: ioannis.georgantopoulos@oabo.inaf.it \\ 2 Institute of Astronomy and Astrophysics, National Observatory of Athens, I. Metaxa \& V. Pavlou str., Palaia Penteli, 15236 Athens, \\ Greece \\ 3 Max-Planck-Institut für Extraterrestrische Physik, Giessenbachstraße, 85748 Garching, Germany
}

Received 28 July 2010 / Accepted 2 September 2010

\begin{abstract}
Recent sub-mm CO molecular line observations of nearby ultra-luminous IRAS galaxies, (U)LIRGs with $L_{\mathrm{IR}} \geq 10^{11} L_{\odot}$, have shown that exceptionally large gas column densities $\left(N_{\mathrm{H}} \geq 10^{25} \mathrm{~cm}^{-2}\right)$ can be present across some of the very dense gaseous disks that are typically found in these objects. In particular, Papadopoulos et al. proposed a diagnostic for finding such obscured sources using $\mathrm{CO}$ and $\mathrm{HCN}$ molecular lines. Given that these high column densities are expected to absorb any X-ray luminous active galactic nuclei (AGN), yielding Compton-thick sources, we set out to explore whether this can be discerned using X-ray observations. More specifically we examine X-ray spectral observations of 14 sources in their sample, using public Chandra observations $(0.5-10 \mathrm{keV})$ for eleven sources as well as BeppoSAX results (2-100 keV) from the literature for another three sources. Our goal is to find candidate Compton-thick AGN and to check whether the molecular line selection criterion is successful in selecting these systems. X-ray spectroscopy reveals four candidate Compton-thick AGN, of which two fall within the high obscuration region in the molecular line ratio diagnostics. Of the remaining five sources falling into the "high dust obscuration" box, one (Mrk273) is highly obscured $\left(N_{\mathrm{H}} \sim 4 \times 10^{23} \mathrm{~cm}^{-2}\right)$, while in the other four the X-ray emission is most probably associated with star-forming processes rather than an AGN on the basis of their X-ray and mid-infrared properties. Overall, we argue that although this method cannot of course recover all Compton-thick AGN, there are no examples of X-ray luminous AGN inside that region that have low obscuration, suggesting that this method is efficient in finding heavily obscured AGN in dust-enshrouded star-forming galaxies. The above results bear important implications for future joint ALMA and X-ray observations for the detection of Compton-thick AGN.
\end{abstract}

Key words. X-rays: general - X-rays: diffuse background - X-rays: galaxies - galaxies: active - submillimeter: galaxies infrared: galaxies

\section{Introduction}

X-ray surveys provide the most efficient tool for identifying active galactic nuclei (AGN) (Brandt \& Hasinger 2005). This is because the hard X-rays can penetrate large amounts of dust and gas and thus can reveal hidden AGN, which would be very difficult to detect at optical wavelengths. However, even the hardest $\mathrm{X}$-rays cannot easily escape from very large absorbing column densities, $>10^{24} \mathrm{~cm}^{-2}$, (Murphy \& Yaqoob 2009). A column density of a few times $10^{24} \mathrm{~cm}^{-2}$ absorbs X-rays up to $20 \mathrm{keV}$, while for column densities higher than $10^{25} \mathrm{~cm}^{-2}$ practically all $\mathrm{X}$-ray photons are attenuated. Such immense column densities $\left(>10^{24} \mathrm{~cm}^{-2}\right)$ correspond to the so-called Compton-thick AGN, where Compton scattering becomes the dominant attenuation process. In these cases reflected emission from the backside of the obscuring screen in form of a torus remains the only evidence of the AGN presence in X-rays (Matt et al. 2004).

These extreme column densities could be relatively easily explored in the IR or sub-mm part of the spectrum through their thermal or molecular emission. For example, sub-mm observations have drawn much attention recently as a means of discovering heavily obscured AGN (e.g. Alexander et al. 2005). The discovery of significant dust optical depths even at submillimeter wavelengths (in the case of Arp220; Sakamoto et al. 2008) reveals that such highly obscured systems may not be

* Table 1 is only available at http://www . aanda.org uncommon. Recently, Papadopoulos et al. (2010) (hereafter P10) made the interesting suggestion that observations of suitable molecular lines can reveal cases of very high column densities (and thus possible obscuration) present in some reservoirs of molecular gas in LIRGs. The average molecular gas densities in some of these systems can be so high that if the gas is distributed in a gaseous disk, it can yield extremely high column densities and dust optical depths that suppress the high- $J(6-5)$ $\mathrm{CO}$ line emission to levels well below those expected in starburst galaxies such as (ultra)-luminous IR galaxies, ULIRGs. In these cases the line of sight towards the nucleus would be severely obscured making this source Compton-thick, if an AGN were embedded in the (U)LIRG. A molecular line diagnostic to identify these severely obscured sources was proposed by P10. This helps to distinguish between the degenerate possibilities of high dust obscuration and large reservoirs of non star-forming cool and diffuse gas, whose high- $J(6-5)$ CO lines are genuinely faint rather than immersed to a strong dust continuum. They suggest that high fractions of very dense molecular gas (with $n(\mathrm{H} 2)>$ $\left.10^{4} \mathrm{~cm}^{-3}\right)$ can be traced by a high $\mathrm{HCN}(1-0) / \mathrm{CO}(1-0)$ ratio $(R>0.1)$. Then a low $(R<0.3) \mathrm{CO}(6-5) /(3-2)$ ratio can only be the result of high dust optical depths $\left(\tau_{434 \mu \mathrm{m}} \sim 1\right)$. Consequently there should be a very high probability of Compton-thick AGN for (U)LIRG hosts lying inside their aforementioned selection "window". On the other hand, we note that gaseous disks (in the center of which the AGN are presumably embedded) with 
low average column densities can often yield high column densities along the particular line of sight towards the AGN as a result of their highly turbulent nature (Wada et al. 2009). Thus Compton-thick sources can be expected also outside the dustobscuration selection window, but the vast majority of sources inside it should be Compton-thick. In other words, if molecular gas disks have on average high column densities, as the supression of CO $J=6-5$ emission from their star-forming gas would imply, this will almost certainly be the case for the special line of sight towards the AGN as well, but the reverse does not necessarily hold. Thus the selection window of highly dust-obscured star-forming systems advocated by P10 ought to select almost exclusively Compton-thick AGN, but not all of them.

In this paper we are examining the X-ray properties of the systems in P10, using both literature spectra as well as Chandra archival observations. Our goal is to test whether AGN residing in the P10 box have a higher probability for being classified as Compton-thick sources in X-rays. We adopt $H_{\mathrm{o}}=$ $75 \mathrm{~km} \mathrm{~s}^{-1} \mathrm{Mpc}^{-1}, \Omega_{\mathrm{M}}=0.3, \Omega_{\Lambda}=0.7$ throughout the paper.

\section{Data and analysis}

\subsection{The sample}

Papadopoulos et al. (2010) present molecular line spectra for 20 (U)LIRGs. For six objects there are no HCN observations available so that the $\mathrm{HCN} / \mathrm{CO}$ vs. $\mathrm{CO}(6-5) / \mathrm{CO}(3-2)$ diagnostic cannot be applied. For the remaining 14 objects there are X-ray spectra available either in the literature or in publicly available Chandra observations. Although in many cases the Chandra observations may have been presented elsewhere, we chose to reanalyze the data in order to provide a uniform analysis of the sample. To our knowledge, in six cases X-ray spectral fits to the Chandra data are presented here for the first time: VII Zw 031, IRAS 10565+2448, IRAS 12112+0305, Arp 193, IRAS 172080014, IRAS 23365+3604. However, X-ray luminosities or hardness ratios derived from Chandra observations have been presented before in the literature for some of those (see Table 1). In the cases of Mrk 231, NGC 6240 and NGC 7469, where there are high signal-to-noise spectra in the $2-100 \mathrm{keV}$ band reported in the literature from BeppoSAX, we prefer to present those data instead, as high energy observations can be much more efficient in detecting Compton-thick sources.

\subsection{Spectral fits}

We fit the Chandra data in the $0.5-10 \mathrm{keV}$ band using XSPEC v.12.6 (Arnaud 1996). Initially, we fit an absorbed power-law model to the spectra. For more complex spectra, and judging on the basis of the data residuals from the model fit, we additionally fit a thermal component (Raymond-Smith) to account for X-ray emission from star-forming processes, and/or an $\mathrm{FeK} \alpha$ line around $6.4 \mathrm{keV}$. The $\mathrm{C}$-statistic has been employed (Cash 1979). The errors correspond to the $90 \%$ confidence level, as is customary in X-ray astronomy. The X-ray spectral properties and luminosities are presented in Table 1 . In the Chandra data presented here, an FeK $\alpha$ line has been detected forf the sources IRAS 09320+6134 (see discussion below) and the Seyfert-2 galaxy Mrk 273. An in-depth presentation of the Chandra spectrum of this source can be found in Xia et al. (2002) and Ptak et al. (2003).

We use the following criteria to classify a source as Compton-thick: a) the detection of an absorption turnover, b) a flat X-ray spectrum with $\Gamma \sim 1$ or flatter, suggestive of a reflection-dominted spectrum, or finally c) a high $E W(>0.8 \mathrm{keV})$ FeK $\alpha$ line, indicating a strongly absorbed continuum (see discussion in Matt et al. 2004; Akylas \& Georgantopoulos 2009; Georgantopoulos et al. 2009, for justification and explanation of these diagnostics). On the basis of the above criteria, we consider four Compton-thick sources: IRAS 09320+6134, Mrk 231, Arp 220, and NGC 6240. For Mrk 231 and NGC 6240 the absorption turnovers have been directly detected by BeppoSAX (Braito et al. 2004 and Vignati et al. 1999 respectively) and therefore these can be considered as bona-fide Compton-thick sources. In the case of Arp 220, the spectrum is flat $(\Gamma \sim 1.1)$, but no Fe line can be detected in the Chandra data. However, in the XMM-Newton data (Iwasawa et al. 2005) a high $E W(1.9 \mathrm{keV})$ $\mathrm{FeK} \alpha$ line at $6.7 \mathrm{keV}$ has been clearly detected, rendering this source a highly probable Compton-thick source. The case of IRAS 09320+6134 (UGC 5101) is the most ambiguous one; we detect a flat spectrum $(\Gamma \sim 1.2)$, albeit with a large uncertainty, together with a high $E W(\sim 3.6 \mathrm{keV}) \mathrm{FeK} \alpha$ line. The combination of a flat spectrum with a high $E W \mathrm{FeK} \alpha$ line rather points towards a Compton-thick AGN. The $E W$ derived here is consistent with the $E W$ obtained by Ptak et al. (2003). Imanishi et al. (2003) analyze both the XMM-Newton and the Chandra data and find a considerably lower $E W(\sim 400 \mathrm{eV})$. González-Martin et al. (2009) claim the detection of a mildly Compton-thick AGN with a column density of $N_{\mathrm{H}} \approx 1.4_{-0.04}^{+0.4} \times 10^{24} \mathrm{~cm}^{-2}$. Finally, for IRAS $23365+3604$, the spectrum is flat, but with a large uncertainty. Moreover, there is no evidence for a high- $E W$ FeK $\alpha$ line, therefore the possibility that this source is Compton-thick is weaker.

\subsection{Galaxies or AGN ?}

From Table 1 we see that a large number of sources (with the exception of Mrk 273, IRAS 05189-2524 and the four Comptonthick AGN) are unobscured with column densities $N_{\mathrm{H}}<$ $10^{22} \mathrm{~cm}^{-2}$. It is possible that in a number of these sources the $\mathrm{X}$-ray emission comes from star-forming processes rather than the AGN. In order to explore this issue, we are plotting the observed (uncorrected for obscuration) X-ray luminosity against the far-IR luminosity in Fig. 1. Ranalli et al. (2003) have found a strong correlation between the X-ray and the far-IR luminosity for star-forming galaxies in the local Universe. This implies that sources with an excess of X-ray emission above this relation can be securely associated with AGN. The sources that fall within the Ranalli et al. (2003) relation should be either "normal" galaxies (i.e. without an AGN) or highly obscured (Compton-thick) AGN. From Fig. 1 we see that three sources (IRAS 05189-2524, Mrk 273 and NGC 7469) present X-ray emission above that of "normal" galaxies.

In order to further investigate the energy mechanism of the sources that lie in the window proposed by P10, we use the mid-IR diagnostic diagram of Laurent et al. (2000). This traces the excess of the $5.5 \mu \mathrm{m}$ continuum emission with respect to the $15 \mu \mathrm{m}$ continuum and $6.2 \mu \mathrm{m}$ PAH emission; essentially it is a tracer of hot dust from the AGN, which has a higher $5.5 \mu \mathrm{m} / 15 \mu \mathrm{m}$ flux ratio than the cooler dust associated with HII regions, and low $6.2 \mu \mathrm{m}$ PAH flux. The sources within the P10 box, namely Mrk 273, IRAS 09320+6134, IRAS 10565+2448, Arp 220, IRAS 172080014, IRAS $12112+0305$, IRAS $23365+3604$, show a fractional AGN contribution of $77 \%, 80 \%, 5 \%, 60 \%, 14 \%, 21 \%$ and $22 \%$ respectively, (Armus et al. 2007, and V. Charmandaris private communication). For comparison, we note that the two Compton-thick AGN outside the box (Mrk 231 and NGC 6240) 
Table 2. AGN diagnostics.

\begin{tabular}{cccc}
\hline \hline $\begin{array}{c}\text { Name } \\
(1)\end{array}$ & $\begin{array}{c}\text { X-ray spectrum } \\
(2)\end{array}$ & $\begin{array}{c}\text { X-ray - IR } \\
(3)\end{array}$ & $\begin{array}{c}\text { Mid-IR } \\
(4)\end{array}$ \\
\hline IRAS 09320+6134 & $\checkmark$ & & $80 \%$ \\
IRAS 10565+2448 & & & $5 \%$ \\
IRAS 12112+0305 & & $\checkmark$ & $21 \%$ \\
Mrk 273 & $\checkmark$ & & $77 \%$ \\
Arp 220 & $\checkmark$ & & $60 \%$ \\
IRAS 17208-0014 & & & $14 \%$ \\
IRAS 23365+3604 & & & $22 \%$ \\
\hline
\end{tabular}

Notes. The columns are: (1) Name. (2) AGN based on a flat or absorbed spectrum. (3) AGN based on deviation from the Ranalli et al. (2003) relation. (4) Contribution of an AGN to the MIR according to the Laurent et al. (2000) diagnostic.

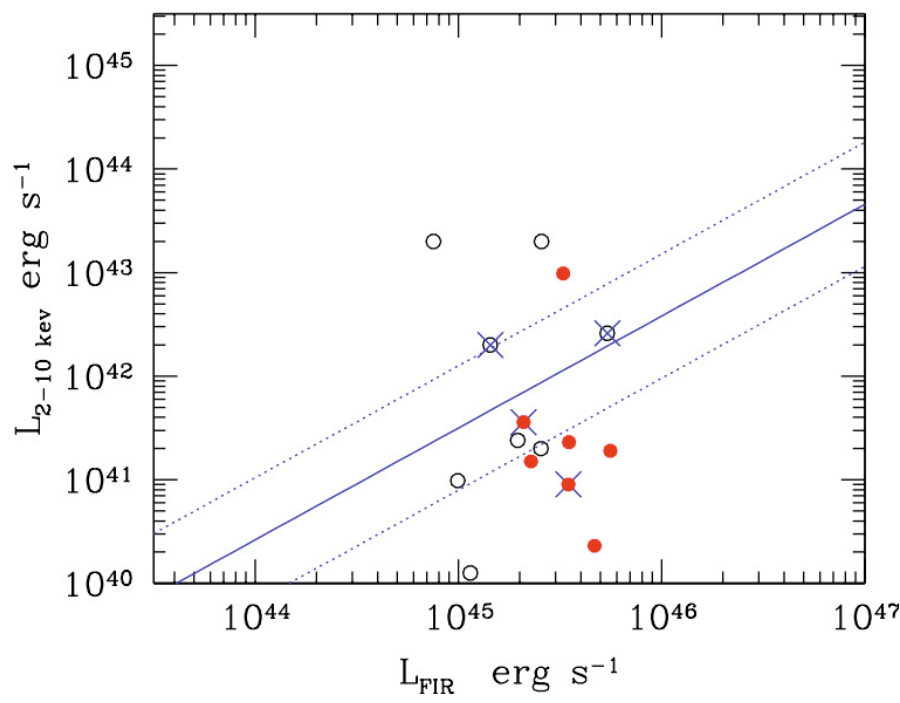

Fig. 1. X-ray $(2-10 \mathrm{keV})$ vs. FIR $(4-100 \mu \mathrm{m})$ relation for our sources. The solid and dotted lines denote the best-fit relation of Ranalli et al. (2003) - and its associated $2 \sigma$ error, for normal galaxies in the local Universe. The red filled circles denote the sources that lie inside the box, while open circles denote those outside the box in the diagnostic diagram of Papadopoulos et al. (2010). Crosses denote the four Compton-thick sources.

show AGN contributions of $95 \%$ and $50 \%$ respectively (Armus et al. 2007).

In Table 2 we summarize the results of the AGN diagnostics used to examine the nature of the sources in the P10 box. There is an agreement between the mid-IR and the X-ray diagnostics which find that $3 / 7$ sources host an AGN, if we use a $50 \%$ as a threshold for the Laurent et al. (2000) diagnostic. We note that one source (IRAS 12112+0305) has been observed with the VLA (Nagar et al. 2003). This source, which is optically classified as a LINER, appears to show a radio core at $15 \mathrm{GHz}$ with a radio power of $\log P \approx 22.6 \mathrm{~W} \mathrm{~Hz}^{-1}$. Therefore, the possibility that this source hosts a Compton-thick AGN cannot be securely excluded.

\section{Discussion and conclusions}

In Fig. 2 we present the diagnostic diagram of P10. We see that out of the four Compton-thick sources (marked with crosses), two (IRAS 09320+6134 and Arp 220) fall within the "high dust obscuration" window. The other one (NGC 6240) lies very close to the box with a marginally lower $\mathrm{HCN}(1-0) / \mathrm{CO}(1-0)$ ratio.

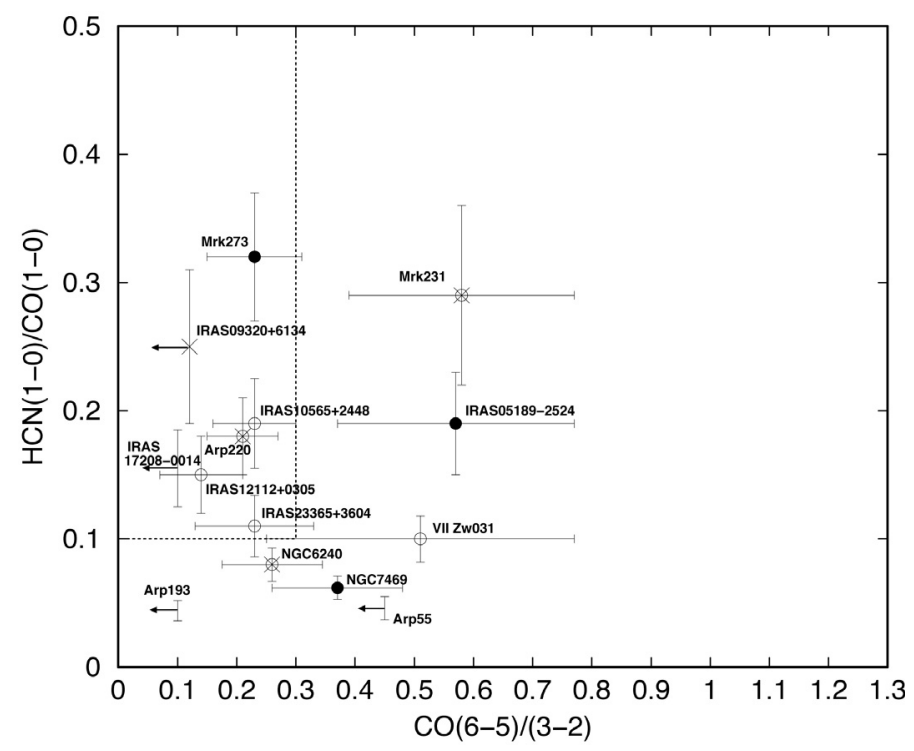

Fig. 2. Diagnostic diagram of Papadopoulos et al. (2010). High HCN(1$0) / \mathrm{CO}(1-0)$ together with low $\mathrm{CO}(6-5) /(3-2)$ ratios (region inside the box) mark the region where high average dust absorption likely surpresses the high- $J$ (in this case $J=6-5$ ) CO emission that is emitted by a heavily dust-enshrouded starburst with large supplies of dense gas (and hence the large $\mathrm{HCN} / \mathrm{CO} J=1-0$ ratio). Filled circles denote the excess X-ray emission sources (i.e. likely AGN) according Fig. 1. Open circles denote sources falling within the Ranalli et al. (2003) relation for normal galaxies (all four $\mathrm{CO}(6-5) /(3-2)$ upper limits fall again within the same relation). Crosses denote the four Compton-thick sources according to X-ray spectroscopy.

The fourth source (Mrk 231) lies away from the box showing highly excited $\mathrm{CO}$ lines and a high $\mathrm{HCN} / \mathrm{CO} J=1-0$ line ratio. This presents no fundamental problem in the Comptonthick selection using molecular lines, because Compton-thick AGN are also expected to lie outside the "window" of the diagnostic diagram of P10. Moreover, this source displays peculiar characteristics compared to the other three Compton-thick sources: although it is a bona-fide Compton-thick source, it is a broad absorption line (BAL) Quasi-Stellar Object (QSO). BAL is a special class of QSOs that show highly ionized gas flowing away from the central source with speeds in some cases exceeding $30000 \mathrm{~km} \mathrm{~s}^{-1}$. Most BAL QSOs present high obscuring columns in X-rays, but their broad-line-regions are still viewed relatively unobscured by dust, probably meaning that their dense obscuring medium is dust-free (e.g. Véron-Cetty \& Véron 2000). It is then reasonable that in the case of Mrk 231 the high $\mathrm{CO}(6-5) / \mathrm{CO}(3-2)$ ratio implies that the $\mathrm{CO}$ emission is not suppressed by dust, despite the presence of a high X-ray obscuring column density.

In addition, we need to know the nature of the remaining sources within the P10 "high dust obscuration" box. Apart from the two Compton-thick AGN there are five more sources presenting characteristically low $\mathrm{CO}(6-5) / \mathrm{CO}(3-2)$ ratios in combination with high HCN/CO $J=1-0$ ones. These are Mrk 273, IRAS 17208-0014, IRAS 10565+2448, IRAS 23365+3604, and IRAS $12112+0305$. Among them only Mrk 273 has a known X-ray luminous, highly obscured $\left(N_{\mathrm{H}} \sim 4 \times 10^{23} \mathrm{~cm}^{-2}\right)$, but not Compton-thick AGN. Thus the total mean column density of a gaseous disk around the AGN that would be responsible for its X-ray obscuration would be $\left\langle N_{\mathrm{H}}(\right.$ disk $\left.)\right\rangle \sim 2 x N_{\mathrm{H}}=$ $8 \times 10^{23} \mathrm{~cm}^{-2}$. On the other hand mean disk column densities as low as $\left\langle N_{\mathrm{H}}(\right.$ disk $\left.)\right\rangle=5.2 \times 10^{24} \mathrm{~cm}^{-2}$ are compatible with 
the observed suppression of the CO 6-5/3-2 ratio in this object, which are 6.5 times higher than the one deduced by the $\mathrm{X}$-ray data. This discrepancy remains considerable, although it is not very large given the totally independent means used to obtain $\left\langle N_{\mathrm{H}}(\right.$ disk $\left.)\right\rangle$ (molecular lines vs. X-rays). However, we note that the substantial difficulty of measuring CO $J=6-5$ fluxes from the Earth with a large telescope like the JCMT will more often yield underestimates rather than overestimates (because pointing errors become substantial with respect to the narrow beams used). This could tend to make molecular line-deduced and X-ray-deduced hydrogen column densities more compatible in cases like Mrk 273.

The X-ray emission of the remaining four sources in the box is probably dominated by star-forming processes according to their X-ray spectra, the $L_{X}-L_{\text {FIR }}$ relation and the MIR diagnostics of Laurent et al. (2000). This could explain why the sources do not appear as Compton-thick in X-ray wavelengths, because the origin of the X-rays is not a potentially deeply buried AGN but stellar. Thus these sources are not useful in deducing mean column densities of gaseous disks around AGN (and could be indeed found inside the selection "window" of P10 without invalidating its utility).

As a consistency check it is interesting to discuss the Si $9.7 \mu$ m properties of the objects within the P10 box, because it is clearly impossible that these have substantial dust optical depths in short sub-mm wavelengths as suggested by P10, while at the same time they exhibit modest or low $\mathrm{Si}(9.7 \mu \mathrm{m})$ optical depths (e.g. Shi et al. 2006). Therefore the highly obscured objects according to P10 should present substantial Si optical depths. There are Spitzer-IRS spectroscopic observations for four sources in the P10 box: Mrk 273, IRAS 09320+6134, Arp 220 and IRAS 12112+0305 (Armus et al. 2007). Their Si $(6.7 \mu \mathrm{m})$ optical depths are high: $1.8 \pm 0.4,1.6 \pm 0.3,3.3 \pm 0.2$ and $1.3 \pm 0.3$ respectively. This clearly suggests an association of all the sources in the P10 box with optically-thick systems at $9.7 \mu \mathrm{m}$.

In conclusion, our results show that the diagnostic proposed by P10 offers a potentially useful tool for the detection of highly obscured X-ray luminous AGN. This bears important implications for future facilities such as ALMA. However, our work identified two caveats. First, the AGN inside the P10 box are heavily obscured but not necessarily Compton-thick, as the case of Mrk273 implies. Second, there can be large contaminations by powerful star-forming systems. Additional X-ray spectral observations might be necessary in order to sift those out. More molecular line data, ranging from low to high- $J$ CO lines, as well as low-frequency (and thus unaffected by dust exinction) density indicators, such as low-to-modest- $J$ HCN and CS lines of nearby (U)LIRGs are needed (along with X-ray data) to verify (or not) this picture in local (U)LIRGs that are known to have X-ray luminous AGN. The now superbly operating Herschel Space Observatory which can detect numerous high-J CO lines in local (U)LIRGs (e.g. van der Werf et al. 2010) in combination with ground based low-J $\mathrm{CO}$ and $\mathrm{HCN}$, CS observations presents an excellent opportunity to do so. The large (U)LIRG sample that is now observed as part of the Herschel Key Project HerCULES, in combination with the extensive ground-based low-frequency $\mathrm{CO}$ and $\mathrm{HCN}$ survey will be ideal for such studies.

Acknowledgements. We thank the anonymous referee for many useful comments and suggestions, which helped to substantially improve this paper. I.G. acknowledges the receipt of a Marie Curie fellowship FP7-PEOPLE-IEF-2008 Prop. 235285. We thank P. P. Papadopoulos for reading the manuscript and providing useful comments and suggestions. The Chandra data were taken from the Chandra Data Archive at the Chandra X-ray Center.

\section{References}

Akylas, A., \& Georgantopoulos, I. 2009, A\&A, 500, 999

Alexander, D. M., Smail, I., Bauer, F. E., et al. 2005, Nature, 434, 738

Armus, L., Charmandaris, V., Bernard-Salas, J., et al. 2007, ApJ, 656, 148 Arnaud, K. A. 1996, Astronomical Data Analysis Software and Systems V, ed. G. Jacoby, \& J. Barnes, ASP Conf. Ser., 101, 17

Braito, V., Della Ceca, R., Piconcelli, E., et al. 2004, A\&A, 420, 79

Brandt, W. N., \& Hasinger, G. 2005, ARA\&A, 43, 827

Cash, W. 1979, ApJ, 228, 939

Clements, D. L., McDowell, J. C., Shaked, S., et al. 2002, ApJ, 581, 974

Dadina, M. 2007, A\&A, 461, 1209

Georgantopoulos, I., Akylas, A., Georgakakis, A., \& Rowan-Robinson, M. 2009, A\&A, 507, 747

González-Martin, O., Masegosa, J., Marquez, M., Guainazzi, M., \& Jimnez-Bailn, E. 2009, A\&A, 506, 1107

Imanishi, M., Terashima, Y., Anabuki, N., \& Nakagawa, T. 2003, ApJ, 596, L167 Iwasawa, K., Sanders, D. B., Evans, A. S., et al. 2005, MNRAS, 357, 565 Iwasawa, K., Sanders, D. B., Evans, A. S., et al. 2009, ApJ, 695, L103 Laurent, O., Mirabel, I. F., Charmandaris, V., et al. 2000, A\&A, 359, 887 Matt, G., Bianchi, S., Guainazzi, M., \& Molendi, S. 2004, A\&A, 421, 473 Murphy, K. D., \& Yaqoob, T. 2009, MNRAS, 397, 1549

Nagar, N. M., Wilson, A. S., Falcke, H., Veilleux, S., \& Maiolino, R. 2003, A\&A, 409, 115

Papadopoulos, P. P., van der Werf, P., Isaak, K., \& Xilouris, E. M. 2010, ApJ, 715,775

Ptak, A., Heckman, T., Levenson, N. A., Weaver, K., \& Strickland, D. 2003, ApJ, 592, 782

Ranalli, P., Comastri, A., \& Setti, G. 2003, A\&A, 399, 39

Sakamoto, K., Wang, J., Wiedner, M. C., et al. 2008, ApJ, 684, 957

Shi, Y., Rieke, G. H., Hines, D. C., et al. 2006, ApJ, 653, 127

Teng, S. H., Wilson, A. S., Veilleux, S., et al. 2005, ApJ, 633, 664 van der Werf, P. P., Isaak, K. G., Meijerink, R., et al. 2010, A\&A, 518, L42 Veilleux, S., Sanders, D. B., \& Kim, D. C. 1999, ApJ, 522, 139 Véron-Cetty, M. P., \& Véron, P. 2000, A\&ARv, 10, 81 Vignati, P., Molendi, S., Matt, G., et al. 1999, A\&A, 349, L57 Wada, K., Papadopoulos, P. P., \& Spaans, M. 2009, ApJ, 702, 63 Xia, X. Y., Xue, S. J., Mao, S., et al. 2002, ApJ, 564, 196 
Table 1. X-ray properties.

\begin{tabular}{|c|c|c|c|c|c|c|c|c|c|c|}
\hline $\begin{array}{l}\text { Name } \\
\text { (1) }\end{array}$ & $\begin{array}{l}\text { ObsID } \\
(2)\end{array}$ & $\begin{array}{l}\mathrm{N}_{\mathrm{H}} \\
(3)\end{array}$ & $\begin{array}{c}\Gamma \\
(4)\end{array}$ & $\begin{array}{l}k T \\
(5)\end{array}$ & $\begin{array}{c}E \\
(6)\end{array}$ & $\begin{array}{l}E W \\
(7)\end{array}$ & $\begin{array}{c}\log L_{X} \\
\quad(8)\end{array}$ & $\begin{array}{c}\log L_{\mathrm{FIR}} \\
\text { (9) }\end{array}$ & $\begin{array}{l}\text { class. } \\
(10)\end{array}$ & $\begin{array}{l}\text { Ref. } \\
\text { (11) }\end{array}$ \\
\hline VII Zw031 & 7887 & $<0.12$ & $1.92^{+0.47}$ & - & & & 41.38 & 11.71 & - & $\mathrm{x}$ \\
\hline 05189-2524 & 2034 & $7.6_{-0.94}^{+0.85}$ & $1.75_{-0.18}^{+0.32}$ & $0.07_{-0.07}^{+0.07}$ & & & 43.30 & 11.83 & Sy2 & $\mathrm{x}, \mathrm{a}$ \\
\hline Arp55 & 6857 & $<0.92$ & $\begin{array}{l}2.65_{-0.83}^{+5.23} \\
\text {. }\end{array}$ & -0.11 & & & 40.10 & 11.48 & - & $\mathrm{x}, \mathrm{b}$ \\
\hline $09320+6134 \dagger$ & 2033 & $<0.3$ & $1.17^{-0.83}$ & - & $6.76 \pm 0.04$ & $3500^{+1500}$ & 41.56 & 11.74 & $\mathrm{~L}$ & $\mathrm{x}, \mathrm{a}, \mathrm{b}$ \\
\hline $10565+2448$ & 3952 & $0.18_{-0.08}^{+0.08}$ & $2.13^{-0.21}-24$ & - & & & 41.19 & 11.78 & - & $\mathrm{x}, \mathrm{d}$ \\
\hline $12112+0305$ & 4934 & $<1.2^{-0.08}$ & $2.78^{+0.24}+.21$ & - & & & 40.36 & 12.09 & $\mathrm{~L}$ & $\mathrm{x}, \mathrm{d}$ \\
\hline Mrk231† & - & $273^{+100}$ & $2.95^{-0.99}$ & & $6.39^{+0.17}$ & $180^{+170}$ & 42.41 & 12.15 & QSO & $\mathrm{e}, \mathrm{h}, \mathrm{a}$ \\
\hline Arp193 & 7811 & $\begin{array}{l}-68 \\
0.10_{-010}^{+0.08}\end{array}$ & $1.71_{-0.36}^{+0.16}$ & - & & & 40.99 & 11.41 & - & $\mathrm{X}$ \\
\hline Mrk273 & 809 & $46.7^{-0.10}$ & 2. & $0.96^{+0.05}$ & $6.59 \pm 0.04$ & $220^{+120}$ & 42.99 & 11.93 & Sy2 & $\mathrm{x}, \mathrm{f}, \mathrm{a}$ \\
\hline Arp220† & 869 & $0.43^{-5.5 .44}$ & $1.11^{+0.52}$ & $0.92^{-0.07}$ & & & 40.95 & 11.96 & $\mathrm{~L}$ & $\mathrm{x}, \mathrm{a}, \mathrm{j}, \mathrm{k}$ \\
\hline NGC $6240 \dagger$ & - & $\begin{array}{c}-0.28 \\
179^{+40}\end{array}$ & $1.98^{+0.25}$ & & $6.50^{+0.15}$ & $1880^{+1590}$ & 42.01 & 11.57 & $\mathrm{~L}$ & $\mathrm{~h}, \mathrm{~g}, \mathrm{a}$ \\
\hline $17208-0014$ & 2035 & $0.13^{-15}+10$ & $1.66^{-0.16}$ & - & & & 41.28 & 12.17 & $\mathrm{HII}$ & $\mathrm{X}$ \\
\hline NGC7469 & - & $<0.03$ & $2.05^{+0.02}$ & & $6.35^{+0.14}$ & $84^{+20}$ & 43.27 & 11.30 & Sy 1.5 & $\mathrm{~h}$ \\
\hline $23365+3604$ & 4115 & $<0.4$ & $1.2_{-043}^{+0.037}$ & - & & & 41.38 & 11.96 & L & $\mathrm{x}, \mathrm{i}$ \\
\hline
\end{tabular}

Notes. The columns are: (1) Name; A † denotes candidate Compton-thick sources. (2) Chandra Observation ID in the case where we performed our own spectral fits. (3) Column density in units of $10^{22} \mathrm{~cm}^{-2}$. (4) Power-law photon index. (5) Temperature of the thermal component in keV. (6) Energy of the $\mathrm{FeK} \alpha$ line in $\mathrm{keV}$. (7) $E W$ of the $\mathrm{FeK} \alpha$ line in eV. (8) Logarithm of the absorbed hard (2-10 keV) X-ray luminosity (erg s ${ }^{-1}$ ). (9) Logarithm of the far-infrared luminosity $(40-100 \mu \mathrm{m})$ in units of solar luminosity. (10) Optical classification according to Veilleux et al. (1999) or the NASA Extragalactic Database. (11) X-ray reference: a. Ptak et al. (2003), b. González-Martin et al. (2009), c. Imanishi et al. (2003) d. Iwasawa et al. (2009) e. Braito et al. (2004) f. Xia et al. (2002) g. Vignati et al. (1999) h. Dadina (2007) i. Teng et al. (2005) j. Clements (2002) k. Iwasawa et al. (2005) x. this work. 